\title{
CONSTRUCCIÓN Y VALIDACIÓN DE UN INSTRUMENTO DIAGNÓSTICO PARA LA REPRESENTACIÓN COGNITIVA DE LA ORIENTACIÓN PSICOPEDAGÓGICA EN GALICIA
}

\author{
CONSTRUCTION AND VALIDATION OF A DIAGNOSTIC INSTRUMENT FOR \\ THE COGNITIVE REPRESENTATION OF THE GUIDANCE IN GALICIA
}

\author{
José Antonio Sarmiento Campos*, Camilo Isaac Ocampo Gómez**, \\ Alfonso Cid Sabucedo*** \\ Universidad de Vigo
}

\section{RESUMEN}

Se presenta el proceso de validación de un instrumento de diagnóstico compuesto por dos elementos: ontología de la orientación psicopedagógica y cuestionario para indagar sobre el modelo común de orientación entre los orientadores educativos de Galicia. En el artículo se muestran las especificaciones técnicas del cuestionario, cuyo índice de consistencia interna es de un Alpha de 0,947. Mediante análisis factorial, de conglomerados y ecuaciones estructurales se identifican dos modelos, uno teórico y otro empírico. La gran afinidad entre ambos da cuenta de la validez de constructo del instrumento. Las conclusiones y la prospectiva del trabajo constituyen la parte final del artículo.

Palabras clave: Orientación psicopedagógica, validez, ontología, ecuaciones estructurales, técnicas estadísticas multivariables.

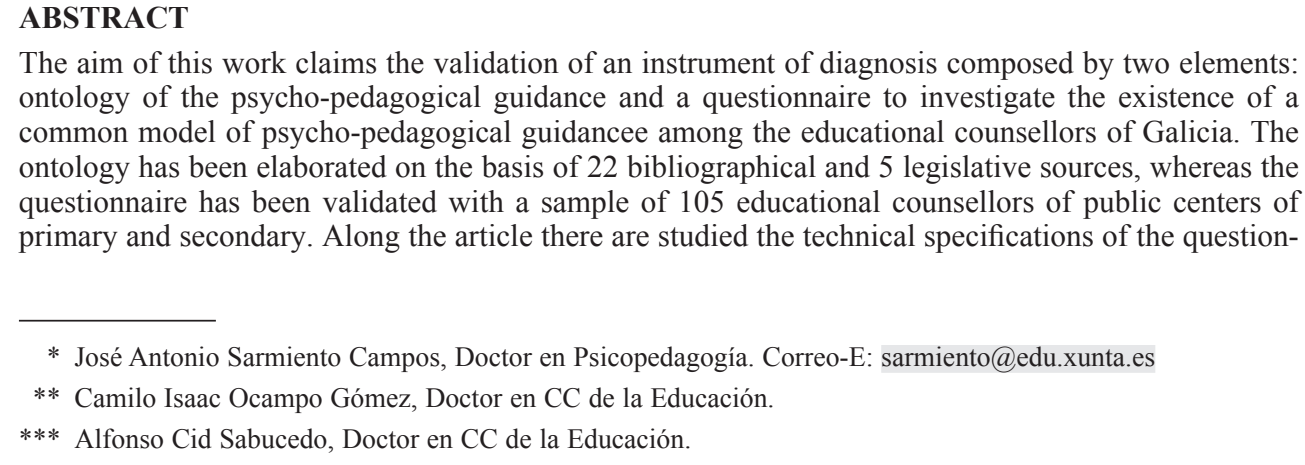

The aim of this work claims the validation of an instrument of diagnosis composed by two elements: ontology of the psycho-pedagogical guidance and a questionnaire to investigate the existence of a common model of psycho-pedagogical guidancee among the educational counsellors of Galicia. The ontology has been elaborated on the basis of 22 bibliographical and 5 legislative sources, whereas the questionnaire has been validated with a sample of 105 educational counsellors of public centers of primary and secondary. Along the article there are studied the technical specifications of the question-

\footnotetext{
* José Antonio Sarmiento Campos, Doctor en Psicopedagogía. Correo-E: sarmiento@edu.xunta.es

** Camilo Isaac Ocampo Gómez, Doctor en CC de la Educación.

*** Alfonso Cid Sabucedo, Doctor en CC de la Educación.
} 
naire. The internal reliability level (Cronbach's alpha) was 0,947 . On the other hand, by means of factor analysis and cluster analysis, several factors are established, as well as its relations, which there form two models, the theoretical and different empirical one. Using structural equations there is verified the adjustment of both models for, finally, to establish the degree of similarity (correlation) between them. The above mentioned contrast concludes in the great affinity between the theoretical model and the empirical model, which realizes of its high construct validity. Finally the conclusions appear, the prospective of the work and of the instrument used.

Key words: Psycho-pedagogical guidance, construct validity, ontology, SEM, factor analysis, cluster analysis, multivariate data analysis, instrument of diagnosis.

\section{Introducción}

El grado de validez de los instrumentos de medida utilizados en las ciencias sociales y más específicamente en el campo psicopedagógico es pieza clave en el progreso de la investigación educativa. La validez en la construcción de los instrumentos para la investigación garantiza la mejora en el corpus teórico de las ciencias a través de su confirmación empírica y muestra hasta qué punto el investigador va por buen camino. Además, el objetivo de trabajos como éste y otros de similar naturaleza (Repetto, Beltrán, Garay-Gordovil y Pena, 2006: 213-223) pretende superar realidades como la recogida por destacados investigadores cuando aluden a problemas de dialectos sociales que tornan difícil la comunicación interprofesional (Santana, 2003: 54), además de problemas terminológicos propiciados por la multiplicidad de enfoques y la confluencia de varias disciplinas en la orientación psicopedagógica (Álvarez y Bisquerra, 1996).

El presente trabajo da cuenta del proceso seguido para estimar la validez de constructo del instrumento diagnóstico empleado en una investigación llevada a cabo en la Comunidad Autónoma de Galicia con los orientadores educativos de los centros públicos sobre su concepto de "orientación psicopedagógica".

\section{El procedimiento de validación teórico-empírico. Entre la epistemología y la praxis: ontología de la orientación psicopedagógica}

La teoría que subyace al instrumento que se analiza surge de un constructo teórico, surgido del campo de la Ingeniería del Conocimiento, denominado "ontología de la orientación psicopedagógica" (Sarmiento, 2007), una estructura de representación que pretende dar respuesta a las cuestiones referidas a los términos que conforman el concepto de orientación psicopedagógica, así como a la tipología, número y naturaleza de sus relaciones.

A partir de la ontología, entendida como "especificación explícita y formal sobre una conceptualización consensuada" (Studer, Benjamins y Fensel, 1998: 162) se elabora una "ontología de la orientación psicopedagógica".

El dominio a cubrir por la ontología se limita a la orientación psicopedagógica como disciplina académica y como campo profesional en el ámbito educativo, con los matices propios de su contextualización geográfica en la Comunidad Autónoma de Galicia.

Su utilización, en un principio, se reduce al diseño y construcción de instrumentos de recogida de datos. El que se analizará en este trabajo fue elaborado para la recogida de in- 
formación sobre la representación del modelo común que, sobre orientación psicopedagógica en Galicia, tienen los agentes de la orientación.

Las preguntas a las que procura dar respuesta la ontología se refieren tanto al ámbito académico y teórico de la orientación, como a su dimensión profesional y normativa. La idea original sobre su uso, prevé su utilización por parte de orientadores profesionales, estudiantes y profesorado universitario.

\section{Fuentes teóricas}

La ontología de la orientación psicopedagógica, aquí implementada como estructura formal, toma de diversas fuentes el conocimiento que contiene, en este caso de 22 bibliográficas y 5 documentos legislativos que aparecen recogidos en la tabla 1.

TABLA 1: Fuentes utilizadas en el diseño de la ontología de orientación.

\begin{tabular}{|c|c|}
\hline Título & Autor/es \\
\hline Orientación educativa. Teoría, evaluación e intervención & Alonso Tapia, J \\
\hline Manual de Orientación y tutoría & Álvarez, M. y Bisquerra, R. \\
\hline $\begin{array}{l}\text { Orientación educativa y acción orientadora. Relaciones entre la } \\
\text { teoría y la práctica }\end{array}$ & Álvarez Rojo, V. \\
\hline $\begin{array}{l}\text { Análisis de las competencias profesionales de los orientadores } \\
\text { escolares. }\end{array}$ & Barreira Arias, J. A. \\
\hline Modelos de orientación e intervención psicopedagógica & Bisquerra Alzina, R. (Coord.) \\
\hline $\begin{array}{l}\text { Orientación y comunidad. La responsabilidad social de la } \\
\text { orientación }\end{array}$ & Gordillo, M. V. \\
\hline Orientacion y accion tutorial: de la teoria a la practica. & Galve, J. L. y Ayala, C. L. \\
\hline $\begin{array}{l}\text { Orientación escolar e acción titorial en Galicia (informe do } \\
\text { Consello Escolar de Galicia) }\end{array}$ & ICE-USC \\
\hline $\begin{array}{l}\text { La orientación psicopedagógica. Modelos y estrategias de } \\
\text { intervención }\end{array}$ & Martínez Clares, P. \\
\hline Orientación educativa e intervención psicopedagógica & Repetto Talavera, E. \\
\hline $\begin{array}{l}\text { Modelos de orientación e intervención psicopedagógica. Vol I. } \\
\text { Marco conceptual y metodológico }\end{array}$ & Repetto Talavera, E \\
\hline Manual de asesoramiento y orientación vocacional & Rivas. F. E. \\
\hline Teoría y práctica de la orientación educativa & Rodríguez Espinar, S. (Coord.) \\
\hline Orientación e intervención psicopedagógica & Rodríguez Moreno, M. L. \\
\hline Funciones del orientador en primaria y secundaria & Sampascual, N. L. y Castejón, J. \\
\hline Orientación psicopedagógica y calidad educativa & Sanz Oro, R. \\
\hline
\end{tabular}




\begin{tabular}{|l|l|}
\hline \multicolumn{1}{|c|}{ Título } & \multicolumn{1}{|c|}{ Autor/es } \\
\hline $\begin{array}{l}\text { Orientación educativa e intervención psicopedagógica. } \\
\text { Cambian los tiempos, cambian las responsabilidades } \\
\text { profesionales }\end{array}$ & Santana Vega, L. \\
\hline Evaluación psicopedagógica y orientación educativa & Sobrado, L y Ocampo, C. \\
\hline Servicios de orientación nos centros educativos & Sobrado Fernández, L \\
\hline $\begin{array}{l}\text { Estratexias de orientación psicopedagóxica no ensino } \\
\text { secundario }\end{array}$ & Sobrado Fernández, L \\
\hline Orientación educativa e intervención psicopedagógica & Solé, I. \\
\hline $\begin{array}{l}\text { Orientación e intervención psicopedagógica. Concepto, } \\
\text { modelos, programas y evaluación }\end{array}$ & Vélaz de Medrano, C. \\
\hline \multicolumn{1}{|c|}{ Documentos legislativos } \\
\hline Ley Orgánica 2/2006 de la Educación (LOE) \\
\hline Ley Orgánica 1/1990 de Ordenación General del Sistema Educativo (LOGSE) \\
\hline $\begin{array}{l}\text { Decreto 120/1998 por el que se regula la orientación Educativa y Profesional en la Comunidad } \\
\text { Autónoma de Galicia }\end{array}$ \\
\hline $\begin{array}{l}\text { Orden del 24 de julio de 1998 por la que se establece la organización y funcionamiento de la } \\
\text { orientación educativa y profesional en la Comunidad Autónoma de Galicia. }\end{array}$ \\
\hline $\begin{array}{l}\text { Orden del 27 de diciembre de 2002 por la que se establecen las condiciones y criterios para la } \\
\text { escolarización en centros sostenidos con fondos públicos del alumnado de enseñanzas no } \\
\text { universitarias con necesidades educativas especiales }\end{array}$ \\
\hline
\end{tabular}

Finalmente, y después de extraer la información aportada por las citadas fuentes, la ontología de la orientación psicopedagógica resultante aparece conformada por 44 clases (términos) distribuidas en 4 niveles jerárquicos, 107 atributos (características) y 423 instancias (casos concretos de objetos reales) que, relacionados entre sí, constituyen la teoría de partida en la construcción del cuestionario (Sarmiento, 2007: 312).

\section{Método}

\section{Muestra}

Con el fin de garantizar la suficiencia, o cantidad de elementos requeridos para asegurar un nivel de confianza deseado, y representatividad, es decir, que los elementos seleccionados posean las características que identifican al universo (Padua, 1987), fueron selecciona$\operatorname{dos}^{1}$ aleatoriamente y sin reposición 200 centros educativos de los 949 que componían, en

1. El proceso de muestreo fue realizado durante el mes de septiembre de 2005, prolongándose la recogida de datos hasta diciembre de ese mismo año. 
su momento, la población y, consecuentemente, fue invitado a responder al cuestionario ${ }^{2}$ el jefe del departamento de orientación. Finalmente respondieron a la invitación 133 orientadores. De todos los cuestionarios cubiertos, 18 no fueron válidos, por lo que la muestra quedó fijada en 105 respuestas, lo que garantizaba, en el peor de los casos, un valor de significación del 95\% $(\mathrm{z}=1,06)$, con un error muestral de 0,5 . A través del análisis de los datos proporcionados por los 105 orientadores se pudieron establecer las especificaciones técnicas del cuestionario, fiabilidad y validez.

\section{El cuestionario}

No obstante, la necesidad de elegir una muestra de las clases que conforman la ontología de la orientación, y no la totalidad, deriva de su elevado número ${ }^{3}$ y de la imposibilidad práctica de someterlas todas a la valoración de los orientadores. De ahí que se proceda a una selección mediante la aplicación de la técnica Delphi.

Para el panel de expertos fueron invitados tres profesores universitarios de las universidades gallegas del Área de Métodos de Investigación y Diagnóstico en Educación y dos profesores del Cuerpo de Profesores de Educación Secundaria de la especialidad de Psicología y Pedagogía pertenecientes a los Equipos de Orientación Específicos, todos ellos expertos en la temática aquí abordada ${ }^{4}$.

Finalmente, las cuestiones seleccionadas mediante consenso entre los participantes fueron agrupadas en los siguientes cinco niveles:

- Orientación psicopedagógica.

- Intervención psicopedagógica.

- Contextos.

- Agentes.

- Ámbitos de intervención.

La tabla 2 muestra los 25 items $^{5}$ que aparecen en la versión definitiva del cuestionario, ubicado finalmente en Internet, así como el nivel jerárquico al que pertenecen.

A los sujetos participantes en la investigación se les pidió que valorasen de 1 a 10 (1 valor más bajo y 10 valor más alto) la importancia de cada uno de los 25 elementos orientadores, desde su perspectiva y experiencia teórica y práctica.

2. http://www.ophiusa.es/tesis/cuestionarioI.htm

3. La ontología de la orientación psicopedagógica se compone de 44 clases.

4. Los cinco componentes poseen el grado de doctor.

5. No se recogen las variables de clasificación. 
TABLA 2: Items del cuestionario, variable asociada y nivel jerárquico de pertenencia en la ontología

\begin{tabular}{|c|c|}
\hline Cuestión (variable) & Nivel jerárquico \\
\hline Evaluación psicopedagógica. (V1) & Orientación psicopedagógica \\
\hline $\begin{array}{l}\text { Adquisición de concocimientos en materia de orientación } \\
\text { psicopedagógica. (V2) }\end{array}$ & Orientación psicopedagógica \\
\hline Dominio de modelos teóricos. (V3) & Orientación psicopedagógica \\
\hline Manejo de modelos de intervención. (V4) & Orientación psicopedagógica \\
\hline Aplicación de los principios de la orientación. (V5) & Orientación psicopedagógica \\
\hline Tratamiento de la atención a la diversidad. (V6) & Ámbitos \\
\hline Prevención y desarrollo humano. (V7) & Ámbitos \\
\hline Atención a los procesos de enseñanza-aprendizaje. (V8) & Ámbitos \\
\hline Desarrollo de la carrera. (V9) & Ámbitos \\
\hline Asesoramiento y formación. (V10) & Ámbitos \\
\hline Gestión en la orientación. (V11) & Intervención psicopedagógica \\
\hline Funciones teóricas e institucionales. (V12) & Orientación psicopedagógica \\
\hline Desarrollo profesional. (V13) & Intervención psicopedagógica \\
\hline Disposición y manejo de recursos. (V14) & Intervención psicopedagógica \\
\hline Dominio de estrategias orientadoras. (V15) & Intervención psicopedagógica \\
\hline $\begin{array}{l}\text { Concepto del sistema escolar como contexto de la orientación. } \\
\text { (V16) }\end{array}$ & Contextos \\
\hline $\begin{array}{l}\text { Consideración de los medios comunitarios como contexto de la } \\
\text { orientación. (V17) }\end{array}$ & Contextos \\
\hline $\begin{array}{l}\text { Enfoque de las organizaciones como contexto de la orientación. } \\
\text { (V18) }\end{array}$ & Contextos \\
\hline Consideración de la familia como contexto de la orientación. (V19) & Contextos \\
\hline Múltiples personas como agentes de la orientación. (V20) & Agentes \\
\hline Información actualizada como competencia orientadora. (V21) & Agentes \\
\hline Dominio de técnicas como competencia orientadora. (V22) & Agentes \\
\hline $\begin{array}{l}\text { Estar en posesión de actitudes adecuadas como competencia } \\
\text { orientadora. (V23) }\end{array}$ & Agentes \\
\hline Diseño, aplicación y evaluación de programas orientadores. (V24) & Intervención psicopedagógica \\
\hline Actuación conforme un código ético-profesional. (V25) & Intervención psicopedagógica \\
\hline
\end{tabular}




\section{Resultados}

\section{Especificaciones técnicas: fiabilidad y validez}

La fiabilidad pone en relación las puntuaciones verdaderas de un instrumento con las puntuaciones falibles (puntuaciones más error), entendiendo que en las verdaderas sólo queda recogida la diferencia real de la característica medida (Nunnally, 1987). Para el cálculo estadístico del coeficiente de fiabilidad del cuestionario, como índice de consistencia interna, se ha empleado el modelo alfa, implementado en el paquete informático SPSS 14, que valora la consistencia interna de la escala a partir de la correlación inter-elementos promedio. El resultado obtenido se muestra en la tabla 3.

Tabla 3: Coeficiente de fiabilidad por el método de Alpha de Cronbach.

\begin{tabular}{|c|c|c|}
\hline Casos válidos & N $^{\circ}$ de ítems & Alpha de Cronbach \\
\hline 105 & 25 & 0,947 \\
\hline
\end{tabular}

Según los criterios para la interpretación señalados por Pardo y Ruiz (2002: 598), en el sentido de considerar meritorios los valores por encima de 0,8 y excelentes los que superan 0,9, los resultados obtenidos para el cuestionario corresponden a esta segunda categoría. No obstante cuando aplicamos la misma prueba estadística a cada una de las subescalas teóricas, el índice obtenido varia entre discreto y bueno $(0,55$ subescala contextos, 0,6 subescala agentes, 0,65 subescala orientación psicopedagógica, 0,76 subescala intervención psicopedagógica y 0,775 subescala ámbitos), lo que, como se verá posteriormente, representa la diferencia entre la realidad teórica y empírica.

Se podría afirmar que existe una creencia generalizada, ubicada dentro del imaginario social, de lo qué podría abarcar un concepto como la orientación psicopedagógica, pero al pretender ahondar y matizar existen múltiples aspectos en los que probablemente existiría desacuerdo o desconocimiento. Ese tipo de conceptualizaciones precisas necesitan del acuerdo o consenso sobre sus significados para otorgarles el carácter de útiles y válidos desde un punto de vista científico (Pérez-Gil, Chacón y Moreno, 2000), es decir, necesitan procesos de validación. Y es aquí donde toma su sentido más amplio el concepto de validez de constructo.

Entre los procedimientos o técnicas estadísticas utilizadas para dar cuenta de la validez de un instrumento destacan en gran medida las ecuaciones estructurales, el análisis factorial y el análisis de conglomerados.

\section{Análisis estructurales y normalidad de los datos}

Los estudios de análisis de estructuras son adecuados para llevar a cabo contrastes de teorías, tests de hipótesis o diseños de nuevas teorías. Se puede modelizar sin conocer la estructura de los datos o modelizar según una estructura conocida y compararlos con teorías ya existentes (Lévy y Varela, 2005: 783). No obstante, los resultados han de interpretarse en clave de plausibilidad y temporalidad, no como demostraciones absolutas (Lozano, 2006). 
Pero si el supuesto de normalidad no se cumple, las propiedades de determinados estimadores quedan afectadas, pudiendo resultar distorsionados los contrastes de hipótesis, llevando a conclusiones estadísticamente incorrectas (Batista y Coenders, 2000: 81).

Los datos recogidos a lo largo de la investigación muestran, en su mayoría, una distribución que no cumple con el requisito de normalidad univariante ni, asimismo, multivariante. A pesar de procurar la normalidad de los datos a través de su tranformación mediante las técnicas Box-Cox (García, 1998: 347), en ningún momento fue posible llegar a distribuciones normales en la mayoría de los datos. Debido a lo anterior, y antes de aplicar ninguna técnica de análisis estructural, se optó por una reducción dimensional de las variables a través de su agrupación en los diferentes factores que, por un lado, sugería la teoría inicial residente en la ontología de la orientación psicopedagógica, y por otro lado los que, estadísticamente, sugerían los datos recogidos. Se pretendió la combinación de varias variables observadas para obtener una variable ficticia por bloque de agrupación, que las representara, con la mínima pérdida de información (Pérez, 2005: 489). El proceso anterior, así como el análisis factorial confirmatorio a través de ecuaciones estructurales, se muestra seguidamente.

\section{Análisis factorial confirmatorio. Factores teóricos y factores empíricos}

Para la extracción de los factores presentes en los niveles de la teoría subyacente a la construcción del cuestionario, se ha realizado un análisis factorial con método de extracción de máxima verosimilitud, puesto que por componentes principales sería extraída toda la varianza, sin presentar los errores de medida (Lévy y Varela, 2005: 784), hecho que influiría negativamente en los resultados de los análisis de estructura. Solamente se ha contemplado un factor por cada nivel teórico. Las varianzas extraídas en cada uno de los factores de los cinco niveles se muestran en la tabla 4.

TABLA 4: Niveles teóricos, variables por nivel, varianza explicada por el factor extraído y carga factorial de cada variable

\begin{tabular}{|l|c|c|}
\hline \multicolumn{1}{|c|}{ Nivel factorizado } & Variables presentes & Varianza explicada \\
\hline Orientación psicopedagógica & V1, V2, V3, V4, V5 & $64,912 \%$ \\
\hline Intervención psicopedagógica & V11, V13, V15, V24, V25 & $62,071 \%$ \\
\hline Ámbitos & V6, V7, V8, V9, V10 & $57,644 \%$ \\
\hline Agentes & V20, V21, V22, V23 & $65,151 \%$ \\
\hline Contextos & V16, V17, V18, V19 & $67,606 \%$ \\
\hline
\end{tabular}

A primera vista, parece que el alto porcentaje de varianza explicada por el primer factor extraído de cada uno de los "grupos teóricos", así como las elevadas cargas factoriales de las variables, confirma la correspondencia del instrumento de medida con la teoría en la que se apoyó su elaboración. Veamos si la afirmación anterior se corresponde con los resultados obtenidos a través del análisis estructural. 
El primer paso en la modelización de ecuaciones estructurales consiste, primero, en el desarrollo de un modelo basado en la teoría para, posteriormente, construir el diagrama de relaciones (Hair, Anderson, Tathan y Black, 1999: 643).

Después de la reducción dimensional de las variables a cinco factores comprobamos la significatividad de las covarianzas y correlaciones entre dichos factores, para lo cual se empleó el paquete estadístico AMOS 6.0, cuyos resultados estandarizados aparecen en la figura 1 .

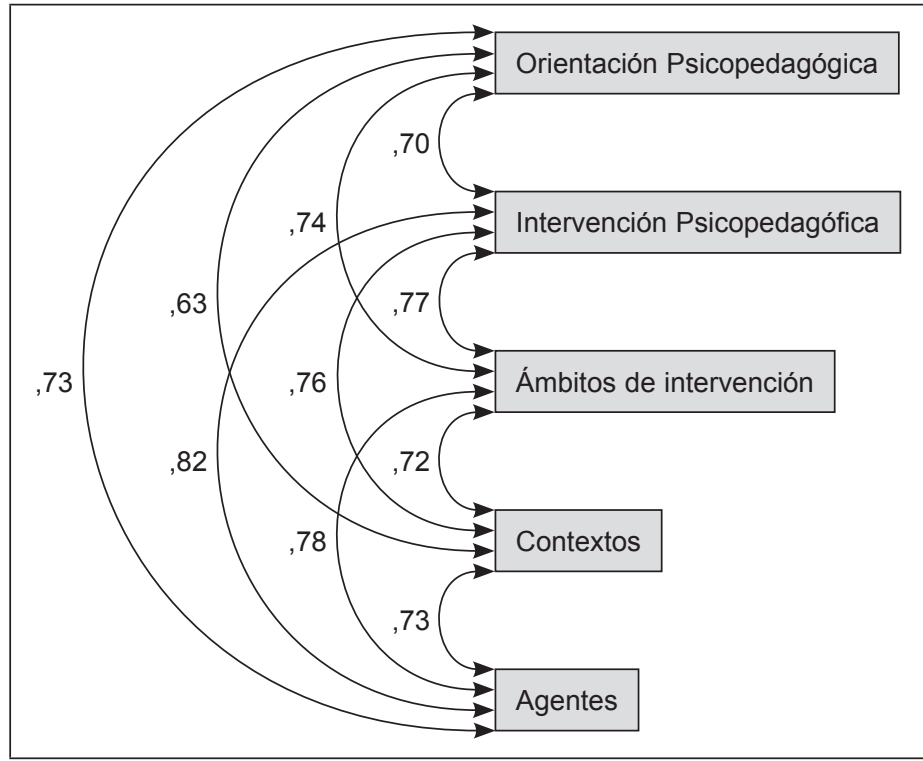

FIGURA 1.

Representación del modelo estructural asociado a los factores teóricos y resultados estandarizados.

Se pueden apreciar los altos índices de correlación entre factores, destacando la relación existente entre el factor que explica la intervención psicopedagógica y el que se refiere a los agentes de la orientación.

Una vez establecido, a través del análisis estructural, el modelo ajustado a la teoría representada en la ontología de la orientación psicopedagógica, y que sirvió de estructura conceptual para la construcción del instrumento de medida, se procedió a explicitar el modelo subyacente en los datos recogidos.

Con el fin de descubrir el número de factores latentes en los datos obtenidos a través del cuestionario, se realizó un análisis de conglomerados de variables de tipo jerárquico, utilizando el método de Ward. La técnica anterior permite la formación de grupos en los que las variables que los componen sean lo más similares posible y los grupos lo más diferentes posible unos de otros (García, 2001: 69). Se pudieron identificar 7 conglomerados de 5, 3, $2,3,5,2$ y 5 variables respectivamente, que darían lugar a 7 factores, 2 más que los presentes en la teoría. 
Como en el caso anterior fue extraído un factor, pero aquí de cada uno de los conglomerados, que explicaba un alto porcentaje de la varianza presente en las variables. También se utilizó el método de extracción de máxima verosimilitud. La varianza explicada por cada factor se recoge en la tabla 5 .

TABLA 5: Niveles empíricos, variables por nivel, varianza explicada por el factor extraído y carga factorial de cada variable

\begin{tabular}{|c|c|c|}
\hline Conglomerado factorizado & Variables presentes & Varianza explicada \\
\hline Conglomerado I & V23, V2, V22, V25, V21 & $68,998 \%$ \\
\hline Conglomerado II & V15, V18, V24 & $77,750 \%$ \\
\hline Conglomerado III & V1, V6 & $66,654 \%$ \\
\hline Conglomerado IV & V3, V4, V5 & $80,249 \%$ \\
\hline ConglomeradoV & V8, V13, V7, V10, V14 & $63,419 \%$ \\
\hline Conglomerado VI & V11, V12 & $68,340 \%$ \\
\hline Conglomerado VII & V20, V9, V16, V17, V18 & $60,212 \%$ \\
\hline
\end{tabular}

Los supuestos básicos del modelo resultante, una vez aplicadas las dos técnicas estadísticas anteriores, aparece representado en la figura 2. Como se puede observar, los siete conglomerados identificados en el análisis de cluster han dado lugar, cada uno de ellos, a un factor:

- Competencias (conglomerado I)

- Intervención y familia (conglomerado II)

- Evaluación desde la diversidad (conglomerado III)

- Praxis teórica (conglomerado IV)

- Ámbitos y recursos (conglomerado V)

- Factor administrativo (conglomerado VI)

- Contextos excepto familia (conglomerado VII)

Siguiendo el modelo resultante del análisis de cluster se establecieron cuatro variables exógenas, dos de primer orden (intervención y marco teórico-práctico) y otras dos de segundo orden (intervención desde marco teórico y contextualización externa).

Para el contraste del modelo expuesto se ha utilizado nuevamente el programa estadístico AMOS 6.0 de SPSS, cuyos índices de ajuste aparecen en la tabla 6.

TABLA 6: Resumen coeficientes e índices de bondad de ajuste del modelo empírico

\begin{tabular}{|c|c|c|c|c|c|c|c|}
\hline$\chi^{2}$ (g.I.) & $\mathbf{p}$ & CFI & GFI & NFI & AGFI & RMSEA & ECVI \\
\hline $8,012(10)$ & 0,628 & 1 & 0,978 & 0,983 & 0,939 & 0 & 0,423 \\
\hline
\end{tabular}


A la vista de los índices anteriores el ajuste del modelo es óptimo, no sólo por el alto valor de $\mathrm{p}$ (lo que nos impide rechazar la hipótesis nula de modelo ajustado), sino por el valor 1 de CFI (índice comparativo de ajuste) y el valor 0 de RMSEA (raíz cuadrada media residual).

Una vez constatado el ajuste del modelo propuesto observemos las relaciones entre las diferentes variables a través de los parámetros estandarizados obtenidos, y que se muestran en la figura 2.

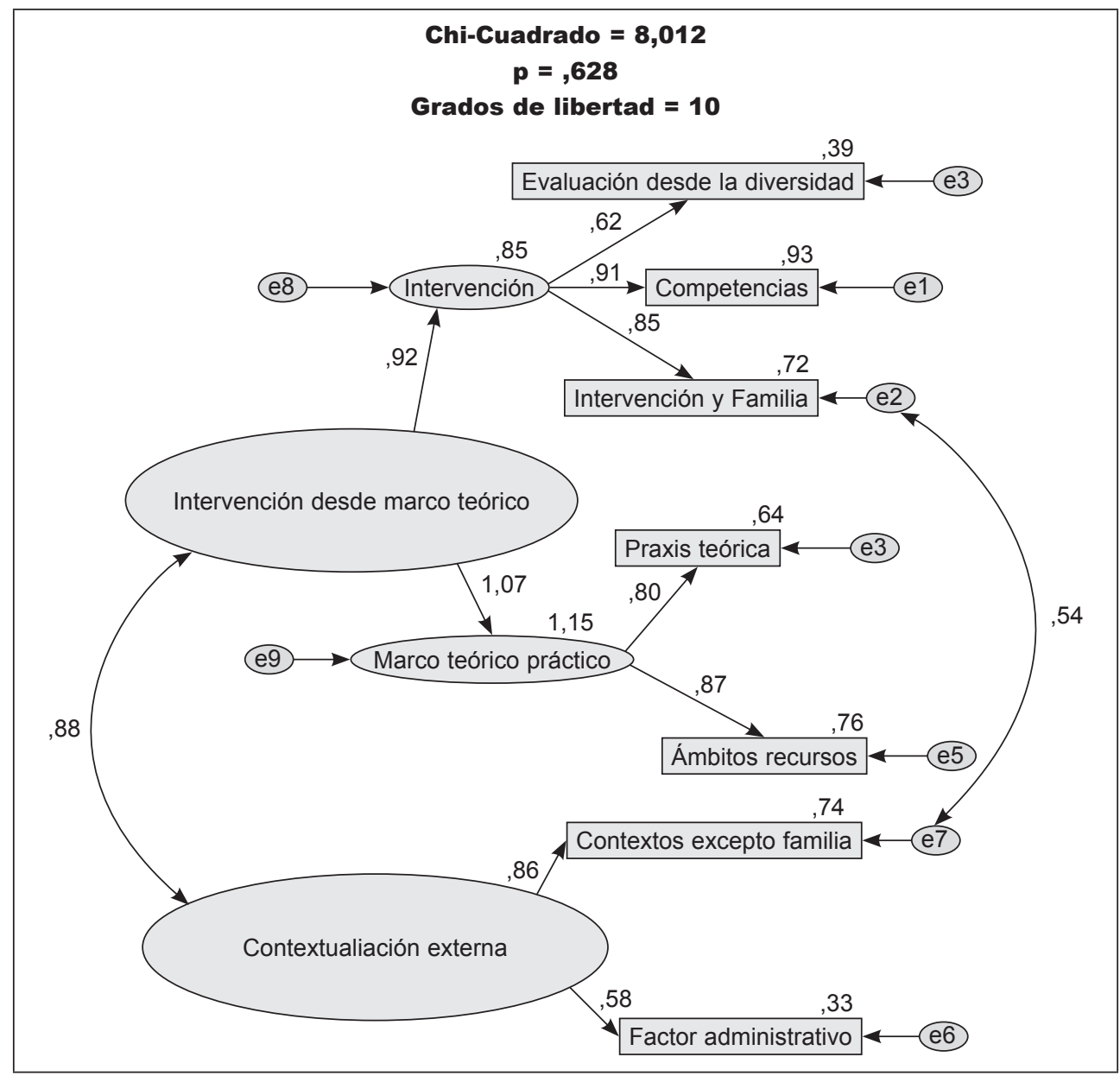

FIGURA 2.

Representación del modelo estructural asociado a los factores empíricos y resultados estandarizados.

Se observan los altos índices de fiabilidad de todos los factores obtenidos de las variables presentes en el cuestionario, a excepción del factor administrativo $(, 33)$ y el referente a la evaluación desde la diversidad $(, 39)$. 
Hasta aquí, se ha demostrado estadísticamente la validez, y siempre a través del análisis de los datos obtenidos, tanto de la teoría que inspiró la creación del cuestionario, como de la estructura conceptual que encerraban dichos datos. Ahora es el momento de demostrar la relación entre ambas para contrastar la validez de constructo del instrumento de medida.

\section{Ajuste entre modelo teórico y modelo empírico}

Con el fin de demostrar el ajuste entre el modelo teórico y el modelo empírico con respecto al instrumento de medida se procedió a la comparación, mediante un análisis de conglomerados, de los factores constitutivos de cada uno de ambos modelos obteniéndose unos resultados que constatan un perfecto acoplamiento entre ellos.

Se han obtenido correlaciones altamente significativas entre todos los elementos de ambos modelos.

El emparejamiento de factores observado en el análisis de conglomerados se establece definitivamente a través de las correlaciones citadas. La matriz de correlaciones muestra la cuasi-identidad entre los siguientes factores:

- Orientación psicopedagógica/Praxis teórica.

- Intervención psicopedagógica/Intervención y familia.

- Ámbitos de intervención/Ámbitos y recursos.

- Contextos/Contextos excepto familia.

- Agentes/Competencias.

Hemos verificado a través de ecuaciones estructurales la validez de los modelos teórico y empírico surgidos de la elaboración del cuestionario y de los datos recogidos a través del mismo. Seguidamente se ha comprobado el ajuste mediante análisis de conglomerado y análisis correlacional de los dos modelos citados, lo que nos lleva a concluir sobre la validez de constructo del instrumento de medida elaborado a partir de la ontología de la orientación psicopedagógica.

\section{Conclusiones}

Habremos de ser cautelosos con las conclusiones, fundamentalmente por dos hechos:

1. La ontología de la orientación psicopedagógica es un constructo dinámico cuya naturaleza no garantiza su estabilidad en el tiempo. No obstante la parte teórica utilizada en la construcción del instrumento de medida se situaba en los niveles más altos de la jerarquía ontológica, menos susceptible de cambios frecuentes.

2. En los modelos propuestos y validados a través de ecuaciones estructurales no se han utilizado las variables representadas en el cuestionario ${ }^{6}$, sino los factores que mejor explicaban la varianza de dichas variables y siempre con referencia a ciertas dimensiones teóricas (a priori) y empíricas (a posteriori).

6. Debido, como ha quedado reflejado, a la falta de normalidad en la distribución de las variables. 
Así pues, y con las referidas cautelas, consideramos garantizada la validez de constructo en el instrumento aquí presentado.

Hemos pretendido probar la citada validez comparando la estructura teórica que sustentó su diseño con la estructura empírica oculta en los datos recogidos. Mediante el contraste estadístico de dos modelos ajustados, el teórico y el empírico creemos haber dado una respuesta positiva a la cuestión anteriormente apuntada. Y todo ello a través de técnicas estadísticas multivariables como el análisis factorial, análisis de conglomerados y modelo de ecuaciones estructurales. Mientras que las dos técnicas analíticas aparecen como fundamentales en la identificación de conjuntos de variables con características afines que pueden dar idea sobre la existencia de constructos ocultos, las ecuaciones estructurales son un ejemplo práctico y empírico del esfuerzo hacia perspectivas holísticas para la resolución de problemas y para validar planteamientos teóricos (Lozano, 2007: 339), que se concretan en estudios prácticos y contextualizados como el que aquí se presenta.

Finalmente el instrumento de diagnóstico ha venido a reflejar las dimensiones que, en un principio aparecían en la teoría: orientación e intervención psicopedagógicas, agentes, contextos y ámbitos. Pero, además, se han podido identificar dos factores de segundo orden que permanecían ocultos, y que se materializan en la intervención psicopedagógica desde el marco teórico y los contextos considerados por los orientadores como externos al centro.

Por lo que respecta a las implicaciones prácticas del presente trabajo, destacar su utilidad en la elaboración de instrumentos de medida fiables dentro de la orientación psicopedagógica a partir de la ontología de la orientación diseñada y en la utilización del cuestionario, así como de los resultados obtenidos para la mejora del proceso formativo de los futuros profesionales de la orientación.

\section{Referencias bibliográficas}

Álvarez, M. y Bisquerra, R. (1996). Manual de orientación y tutoría. Barcelona: Praxis

Batista, J. M. y Coenders, G. (2000). Modelos de ecuaciones estructurales. Madrid: La Muralla/ Hespérides.

García, A. (1998). Estadística aplicada: conceptos básicos. Madrid: UNED.

García, A. (2001). Métodos avanzados de estadística aplicada. Madrid: UNED.

Hair, J., Anderson, R., Tatham, R. y Black W. (1999). Análisis multivariante. Madrid: Prentice Hall.

Lévy, J. P. y Varela, J. (Dirs.) (2003). Análisis Multivariable para las Ciencias Sociales. Madrid: Pearson Educación S.A.

Lozano, S. (2006). Validación de un modelo de medida de la auto-eficacia en la toma de decisiones de la carrera. Revista de investigación educativa. Vol 24, nº 2, 423-442.

Lozano, S. (2007). "Validación de un modelo de medida de las dificultades en los procesos de toma de decisisiones sobre la carrera profesional". Revista de educación. 343. Mayo-agosto 2007, 325351.

Nunnally, J. C. (1987). Teoría psicométrica. México: Trillas.

Padua, J. (1987). Técnicas de investigación aplicadas a las Ciencias Sociales. México D.F.: Fondo de cultura económica.

Pardo, A. y Ruíz, M. A. (2002). SPSS 11. Guía para el análisis de datos. Madrid: McGraw Hill.

Pérez, C. (2005). Métodos estadísticos avanzados con SPSS. Madrid: Thomson. 
Pérez, G. (1994). Investigación cualitativa. Retos e Interrogantes. Madrid: La Muralla.

Pérez-Gil, J. A.; Chacón, S. y Moreno, R. (2000). "Validez de constructo: uso del análisis factorial exploratorio-confirmatorio para obtener evidencias de validez". Psicothema, 12 (2), 442-446.

Repetto, E.; Beltrán, S.; Garay-Gordovil, A. y Pena M. (2006). "Validación del 'Inventario de competencias socioemocionales -importancia y presencia-' (ICS-I; ICS-P) en estudiantes de ciclos formativos y de Universidad". Revista Española de Orientación y Psicopedagogía. Vol. 17, № 2 , $2^{\circ}$ semestre 2006, 213-223.

Santana, L. (2003). Orientación educativa e intervención psicopedagógica. Cambian los tiempos, cambian las responsabilidades profesionales. Madrid: Pirámide.

Sarmiento, J. A. (2007). Modelo común de orientación psicopedagógica en Galicia. Elaboración del IMMT para la representación de conceptualizaciones epistemológicas y subjetivas. Tesis doctoral inédita. Facultad de CC. de la Educación. Universidad de Vigo.

Studer, S.; Benjamins, R. y Fensel, D. (1998). "Knowledge Engineering: Principles and Methods". Data and Knowledge Engineering, 25, 161-197.

Fecha de recepción: 12-02-2008

Fecha de revisión: 17-07-2008

Fecha de aceptación: 19-11-2008 